\title{
Analysis of Switched Capacitor Converter by Fibonacci Sequence for Generating a Staircase Sinusoidal Wave
}

\author{
Wang Lok Do ${ }^{\mathrm{a},}{ }^{*}$, Soranut Kittipanyangam ${ }^{\mathrm{a}}$, Kanji $\mathrm{Abe}^{\mathrm{a}}$, Kei Eguchi $^{\mathrm{a}}$ \\ aFukuoka Institute of Technology, 3-30-1 Wajiro-higashi, Higashi-ku, Fukuoka, 811-0295, Japan \\ *Corresponding Author: dwlkiss88@gmail.com
}

\begin{abstract}
The main purpose of DC-AC converters is to produce AC power by converting DC power. There are many kinds of DC-AC converter with magnetic components. In other words, the components such as inductor or transformer and so forth are included to design and manufacture DC-AC converter in the conventional way until even now. The components make DC-AC converter heavier than DC-AC converter without the magnetic components. In order to overcome this problem, we select one type of DC-AC converter that is the switched capacitor converter operated by Fibonacci sequence for generating an $\mathrm{AC}$ waveform. The validity of the converter about the better efficiency was proved by comparing the conventional ways and the proposed way in the last research. However, the converter in the last research is only for producing a square waveform. Therefore, we analyze whether it is possible to realize that the converter can generate a staircase sine waveform. In order that we implement the above mentioned research, we analyze the converter in a theoretical way. The results of comparing the theoretical analysis and the simulation are illustrated in this paper.
\end{abstract}

Keywords: DC-AC converter, switched capacitor converter, Fibonacci sequence, staircase sine waveform.

\section{Introduction}

There are many types of DC-AC converter in modern society. The purpose of the DC-AC converter is to convert DC power to AC power. Then, the power converted from DC-AC converter is able to be utilized for electronic goods such as home appliance, e-book reader machine, lap-top computer, smart phone and so on. All of appliances introduced above are essential to become much lighter in order to reduce unit price and take much better portability. However, in conventional way until even now, the magnetic components such as inductor or transformer and so forth are used so as to make DC-AC converter. The main aim of the using those magnetic components is to save energy from input power and to transfer the energy, of course it is hard to deny that the magnetic component is the best component in order to do that. However, the magnetic components cause some problems about EMI (electromagnetic interference). For this reason, engineers or designers pays much attention to reduce EMI problems and EMC (electromagnetic compatibility). Another weakness by using the magnetic component is to make the DC-AC converter circuit heavy and bulky. So as to overcome those problems, in 1971, first idea about switched capacitor converter was suggested by Brugler $^{(1)}$. From this suggestion on, many types of switched capacitor DC-AC converter have been suggested and realized by this time; for example, bidirectional inverter ${ }^{(2)}$, ring type converter ${ }^{(3)}$ and switched capacitor voltage multiplier DC-AC inverter ${ }^{(4)}$. In this situation, we made another suggestion that is the design of the switched capacitor DC-AC converter operated by Fibonacci sequence in the last research ${ }^{(5)}$. The proposed converter's better efficiency was proved by comparing the conventional switched capacitor converter and the proposed converter; the conventional switched capacitor converter was suggested in other researches ${ }^{(2-4)}$. However, the analysis and realization of the converter in the last research are only for producing a square waveform. Therefore, we examine and analyze whether it is possible to realize that the converter can generate a staircase sine waveform.

In this paper, we implement the analysis of the switched capacitor converter operated by Fibonacci sequence for producing a staircase sine waveform. In order to do that, we 
analyze the converter in theoretical way. In the step of theoretical analysis, we derive interaction formulas related with all kinds of operation steps for charging and discharging the capacitors in the converter circuit. As the next step, we simulate the converter by SPICE so that we can prove whether the converter is well operated as designed in the theoretical way.

\section{Circuit Configuration}

\subsection{The switched capacitor converter of Fibonacci sequence type}

There are various kinds of type for switched capacitor converter. For example, the types are series-parallel type, ring type and switched-capacitor-voltage-multiplier type and so on ${ }^{(3-5)}$. In many kinds of converter type, it is the Fibonacci sequence type that we select for this paper.

There are two reasons why we select the Fibonacci sequence type. First, we improved that the selected type has higher power efficiency than other conventional types ${ }^{(3-5)}$. Second, the type is possible to make the components of converter circuit minimize. Table 1 complements the second reason.

Fig. 1 shows the switched capacitor converter for DC$\mathrm{AC}$ conversion which was proposed in last research ${ }^{(5)}$. The circuit consists of two blocks. First block for converting DC to $\mathrm{DC}$ is a converter block which is for generating $12 \mathrm{kinds}$ of voltage. Second block for converting DC to AC is a full bridge which is for changing the polarity of the output voltage produced from the converter block. The voltage of each capacitor in the circuit follows the Fibonacci number. The equation (1) shows each voltage of the capacitors in the circuit. That is why we call and name the type of the circuit in Fig 1 the Fibonacci type.

$$
\begin{aligned}
& V_{C n}=V_{C n-1}+V_{C n-2} \text { where } n=\{3,4,5\} \\
& \text { with } V_{c 1}=V_{i n} V_{c 2}=V_{c 1}+V_{i n}
\end{aligned}
$$

Table 1. The number of components for comparison.

\begin{tabular}{|c|c|c|}
\hline Type & Number of switch & Number of capacitor \\
\hline Fibonacci & 17 & 5 \\
\hline Series-parallel $^{(2)}$ & 38 & 12 \\
\hline Ring $^{(3)}$ & 48 & 12 \\
\hline Multistage SCVM $^{(4)}$ & 26 & 8 \\
\hline
\end{tabular}

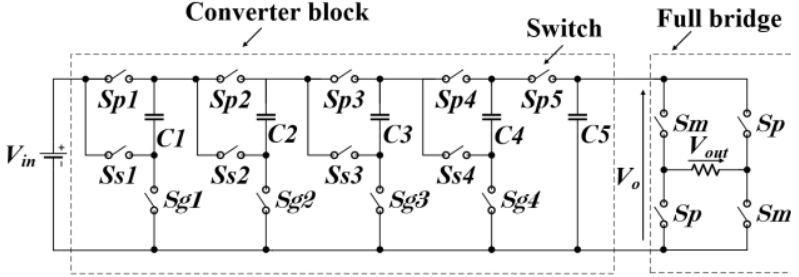

Fig. 1. The switched capacitor converter for DC-AC converter.

\subsection{The way how the converter block is operated}

The converter block in Fig. 1 is operated with the switching rules shown in Tables 2 and 3 which is about how to charge and discharge the capacitors in the converter circuit. In other words, the converter block produces 12 kinds of voltage as the input for the full bridge block by the switching rules. The range of the voltage is from the input voltage to 12 times of the input voltage.

Table 2. Switching rule to charge the capacitors.

\begin{tabular}{|c|c|c|}
\hline Step & Switch to turn on & Switch to turn off \\
\hline State-1 & $S p 1, S g 1, S s 2, S p 3, S g 3$ & Others \\
\hline State-2 & $S s 1, S p 2, S g 2, S s 3, S p 4, S g 4$ & Others \\
\hline
\end{tabular}

Table 3. Switching rule to generate 12 kinds of voltage.

\begin{tabular}{|c|c|c|}
\hline Step & Switch to turn on & Switch to turn off \\
\hline 1 times & $S p 1, S p 2, S p 3, S p 4$ & Others \\
\hline 2 times & $S s 1, S p 2, S p 3, S p 4$ & Others \\
\hline 3 times & $S p 1, S s 2, S p 3, S p 4$ & Others \\
\hline 4 times & $S p 1, S p 2, S s 3, S p 4$ & Others \\
\hline 5 times & $S g 4$ & Others \\
\hline 6 times & $S p 1, S p 2, S p 3, S g 4$ & Others \\
\hline 7 times & $S s 1, S p 2, S p 3, S s 4$ & Others \\
\hline 8 times & $S p 1, S s 2, S p 3, S s 4$ & Others \\
\hline 9 times & $S p 1, S p 2, S s 3, S s 4$ & Others \\
\hline 10 times & $S s 1, S p 2, S s 3, S s 4$ & Others \\
\hline 11 times & $S p 1, S s 2, S s 3, S s 4$ & Others \\
\hline 12 times & $S s 1, S s 2, S s 3, S s 4$ & Others \\
\hline
\end{tabular}

First of all, we are going to explain the switching rules in Tables 2 and 3, the purpose of which is how to charge and discharge the capacitors in the converter. The switching rules is separated as State-1, 2 and 3. State-1 and State-2 are for charging the capacitors in the converter circuit. The sequences of State-1 and State-2 are presented in Fig. 2. In the steps of State-1 and State-2, the input power is charging 


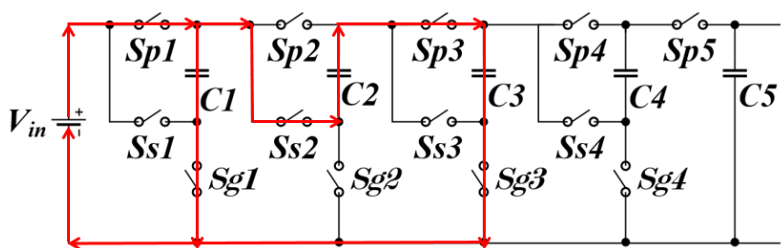

(a) State-1.

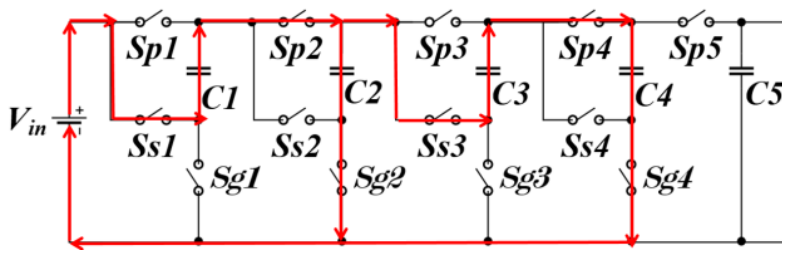

(b) State-2.

Fig. 2. Flow of the current in the converter block.

the capacitors in the converter circuit by the process as follow. 1) In the State-1, the input power is charging $C 1$ in the circuit of Fig. 1 to the level of the input voltage. 2) In the State-2, the input power and the charged $\mathrm{C} 1$ as if a kind of power source are charging $\mathrm{C} 2$ to the level of 2 times of the input voltage. 3) In the State-1 again, the charged $\mathrm{C} 1$ and the charged $\mathrm{C} 2$ are charging $\mathrm{C} 3$ to the level of 3 times input voltage; the input power and $\mathrm{C} 1$ are connected in parallel. 4) In the State-2 again, C4 is charged to the level of 5 times of input voltage by the voltage of $\mathrm{C} 2$ and $\mathrm{C} 3$; the voltage of $\mathrm{C} 2$ is 2 times of the input voltage by 2) and the voltage of $\mathrm{C} 3$ is 3 times of the input voltage by 3 ).

In State-1 and State-2, we implement the charging of the capacitors, the voltage of which follows the Fibonacci sequence. The next step is to generate 12 kinds of voltage. It is possible to produce 12 kinds of voltage by using switching rule shown in Table 3, namely the switching rule of Table 3 means how to connect the switches in order to produce 12 kinds of voltage.

\subsection{The way how the full bridge in the converter is operated}

The purpose of using the full bridge is only for changing the polarity of the current conveyed from the converter block. In order to do this operation, the switches, the name of which is $S_{m}$ and $S_{p}$, should be operated by the switching rule shown in Table 4. Fig. 3 shows the flow of the output current by the switching rule in Table 4.

Table 4. Switching way for the full bridge

\begin{tabular}{|c|c|c|}
\hline Step & Switch to turn on & Switch to turn off \\
\hline Positive & A pair of $S_{m}$ & A pair of $S_{p}$ \\
\hline Negative & A pair of $S_{p}$ & A pair of $S_{m}$ \\
\hline
\end{tabular}

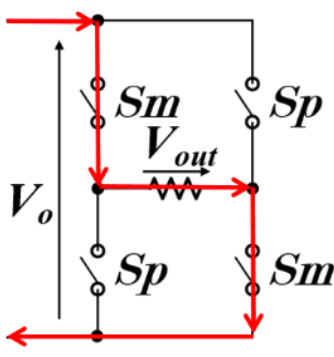

(a) Positive pole.

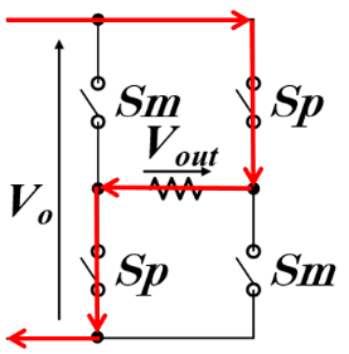

(b) Negative pole.

Fig. 3. Flow of the current in the full bridge.

\section{Theoretical analysis}

\subsection{Equivalent model}

It is necessary to use a four-terminal equivalent model in order to analyze the characteristics the converter circuit. Fig. 4 describes the four-terminal equivalent model for the converter. The equivalent model will be used in next chapter to derive the power efficiency. Thruough the analysis of each equivalent circuit by the switching rules, we can derive the $\mathrm{SC}$ resistance which is called $R s c$. By using the value of $R s c$, it is possible to calculate the power efficiency for the converter in the theretical way.

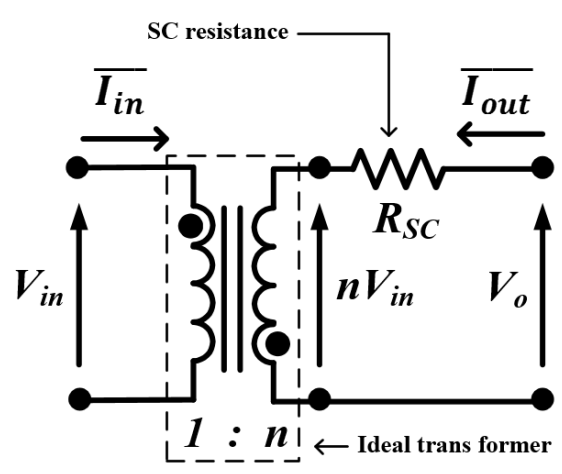

Fig. 4. Four-terminal equivalent model for the converter in this paper. 


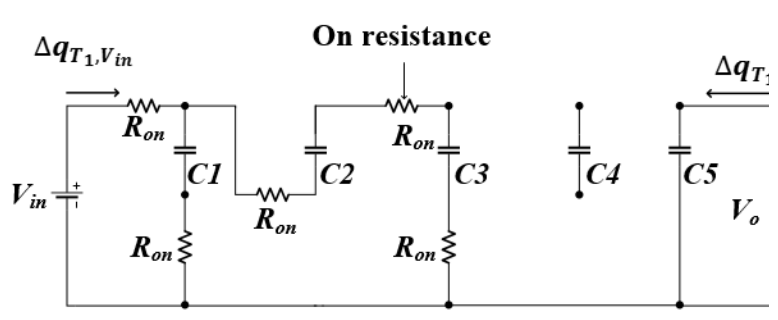

(a) State-1.

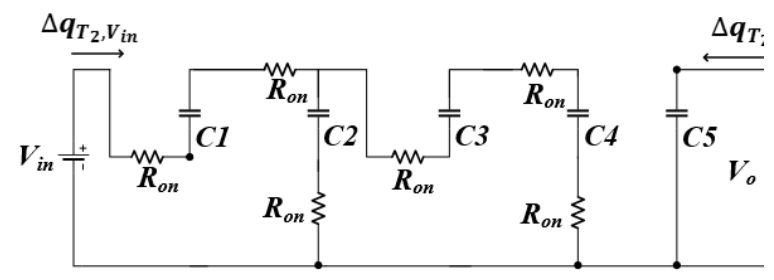

(b) State-2.

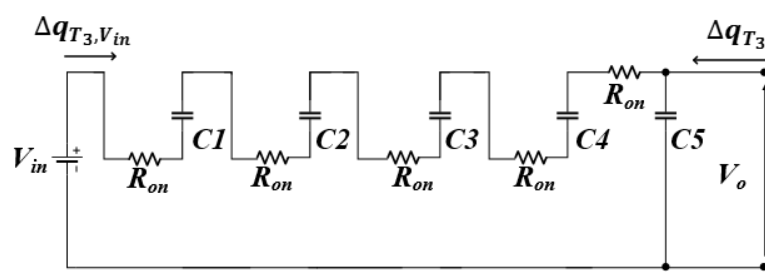

(c) State-3.

Fig. 5. Instantaneous equivalent circuit at each state in 12 times mode

\subsection{SC resistance and efficiency}

We make two assumptions so as to implement the theoretical analysis. First assumption is that the electronic switch such as transistor or MOSFET is an ideal switch with on-resistance $R_{o n}$. Second assumption is that the time constant which is usually called $\tau$ is far beyond all of the clock for the system in this paper.

On Fig. 5, the equivalent circuits at each State with both assumptions are shown; representatively, Fig. 5 is only for 12 times mode.

The converter generates the output voltage of the converter block by the above-mentioned switching rules. And then the produced power is transferred into the full bridge. In this sequence, the analysis of each State is essential to analyze the power efficiency of the converter. In other words, to derive $\mathrm{SC}$ resistance in the equivalent circuit at each State is same to analyze the power efficiency because $\mathrm{SC}$ resistance is only one component that consumes the energy from the input power in the equivalent circuits.

In steady state, all of the differential value of the electric charge in all of the capacitors satisfy equation (2).

$$
\begin{aligned}
& \sum_{i=1}^{3} \Delta q_{T_{i}}^{k} \text { where } T=T_{1}+T_{2}+T_{3} \\
& \text { and } T_{1}=T_{2}=T_{3}=\frac{T}{3} .
\end{aligned}
$$

In equation (2), $\Delta q_{T_{i}}{ }^{k}$ means that the electric charge of the $k$-th capacitor in State- $i$, and $T$ is the period of the system clock for State-1, State-2 and State-3. In this declaration, $T_{i}$ is the $i$-th period of the State- $i$. By using the Kirchhoff's current law in Fig 5, the interaction formula among the factors is derived as follows; the factors are the differential values of electric charges for the input, the output and the capacitors. Equation (3) is for State-1 and Equation (4) is for State-2.

$$
\begin{gathered}
\Delta q_{T_{1}, V_{i n}}=\Delta q_{T_{1}}^{1}-\Delta q_{T_{1}}^{2}, \Delta q_{T_{1}, V_{i n}}=\Delta q_{T_{1}}^{2}, \\
\Delta q_{T_{1}}^{4}=0 \text { and } \Delta q_{T_{1}, V_{o}}=\Delta q_{T_{1}}^{5} \\
\Delta q_{T_{2}, V_{i n}}=-\Delta q_{T_{2}}^{1}, \Delta q_{T_{2}}^{3}=\Delta q_{T_{2}}^{1}+\Delta q_{T_{2}}^{2}, \\
\Delta q_{T_{2}}^{3}=-\Delta q_{T_{2}}^{4} \text { and } \Delta q_{T_{2}, V_{o}}=\Delta q_{T_{2}}^{5}
\end{gathered}
$$

Next step is to derive the equation for State-3. There are 12 kinds of State-3 by the switching rule shown on Table 3. The result of deriving it is given as follows.

For the 1 times the input mode

$$
\begin{gathered}
\Delta q_{T_{3}, V_{i n}}=\Delta q_{T, V_{i n}} \\
\Delta q_{T_{3}}^{1}=\Delta q_{T_{3}}^{2}=\Delta q_{T_{3}}^{3}=\Delta q_{T_{3}}^{4}=0, \\
\text { and } \Delta q_{T_{1}, V_{o}}=\Delta q_{T_{3}}^{5}+\Delta q_{T, V_{i n}}
\end{gathered}
$$

For the 2 times the input mode

$$
\begin{gathered}
\Delta q_{T_{3}, V_{i n}}=-\Delta q_{T_{3}}^{1}, \\
\Delta q_{T_{3}}^{2}=\Delta q_{T_{3}}^{3}=\Delta q_{T_{3}}^{4}=0, \\
\text { and } \Delta q_{T_{3}, V_{o}}=\Delta q_{T_{3}}^{5}+\Delta q_{T_{3}}^{1}
\end{gathered}
$$

For the 3 times the input mode

$$
\begin{gathered}
\Delta q_{T_{3}, V_{i n}}=-\Delta q_{T_{3}}^{2}, \\
\Delta q_{T_{3}}^{1}=\Delta q_{T_{3}}^{3}=\Delta q_{T_{3}}^{4}=0, \\
\text { and } \Delta q_{T_{3}, V_{o}}=\Delta q_{T_{3}}^{5}+\Delta q_{T_{3}}^{2}
\end{gathered}
$$

For the 4 times the input mode

$$
\begin{gathered}
\Delta q_{T_{3}, V_{i n}}=-\Delta q_{T_{3}}^{3}, \\
\Delta q_{T_{3}}^{1}=\Delta q_{T_{3}}^{2}=\Delta q_{T_{3}}^{4}=0, \\
\text { and } \Delta q_{T_{3}, V_{o}}=\Delta q_{T_{3}}^{5}+\Delta q_{T_{3}}^{3}
\end{gathered}
$$

For the 5 times the input mode

$$
\begin{gathered}
\Delta q_{T_{3}, V_{i n}}=0, \\
\Delta q_{T_{3}}^{1}=\Delta q_{T_{3}}^{2}=\Delta q_{T_{3}}^{3}=0, \\
\text { and } \Delta q_{T_{3}, V_{o}}=\Delta q_{T_{3}}^{5}+\Delta q_{T_{3}}^{4}
\end{gathered}
$$


For the 6 times the input mode

$$
\begin{gathered}
\Delta q_{T_{3}, V_{i n}}=-\Delta q_{T_{3}}^{4}, \\
\Delta q_{T_{3}}^{1}=\Delta q_{T_{3}}^{2}=\Delta q_{T_{3}}^{3}=0, \\
\text { and } \Delta q_{T_{3}, V_{o}}=\Delta q_{T_{3}}^{5}+\Delta q_{T_{3}}^{4}
\end{gathered}
$$

For the 7 times the input mode

$$
\begin{gathered}
\Delta q_{T_{3}, V_{i n}}=-\Delta q_{T_{3}}^{1}, \\
\Delta q_{T_{3}}^{2}=\Delta q_{T_{3}}^{3}=0, \\
\text { and } \Delta q_{T_{3}, V_{o}}=\Delta q_{T_{3}}^{5}+\Delta q_{T_{3}}^{1}
\end{gathered}
$$

For the 8 times the input mode

$$
\begin{gathered}
\Delta q_{T_{3}, V_{i n}}=-\Delta q_{T_{3}}^{2}, \\
\Delta q_{T_{3}}^{1}=\Delta q_{T_{3}}^{3}=0, \\
\text { and } \Delta q_{T_{3}, V_{o}}=\Delta q_{T_{3}}^{5}+\Delta q_{T_{3}}^{2}
\end{gathered}
$$

For the 9 times the input mode

$$
\begin{gathered}
\Delta q_{T_{3}, V_{i n}}=-\Delta q_{T_{3}}^{1}, \\
\Delta q_{T_{3}}^{1}=\Delta q_{T_{3}}^{2}=0, \\
\text { and } \Delta q_{T_{3}, V_{o}}=\Delta q_{T_{3}}^{5}+\Delta q_{T_{3}}^{1}
\end{gathered}
$$

For the 10 times the input mode

$$
\begin{gathered}
\Delta q_{T_{3}, V_{i n}}=-\Delta q_{T_{3}}^{1}, \\
\Delta q_{T_{3}}^{2}=0, \\
\text { and } \Delta q_{T_{3}, V_{o}}=\Delta q_{T_{3}}^{5}+\Delta q_{T_{3}}^{1}
\end{gathered}
$$

For the 11 times the input mode

$$
\begin{gathered}
\Delta q_{T_{3}, V_{i n}}=-\Delta q_{T_{3}}^{2}, \\
\Delta q_{T_{3}}^{1}=0,
\end{gathered}
$$$$
\text { and } \Delta q_{T_{3}, V_{o}}=\Delta q_{T_{3}}^{5}+\Delta q_{T_{3}}^{2}
$$

For the 12 times the input mode

$$
\begin{gathered}
\Delta q_{T_{3}, V_{i n}}=-\Delta q_{T_{3}}^{1}, \\
\Delta q_{T_{3}}^{1}=\Delta q_{T_{3}}^{2}=\Delta q_{T_{3}}^{3}=\Delta q_{T_{3}}^{4}, \\
\text { and } \Delta q_{T_{1}, V_{o}}=\Delta q_{T_{3}}^{5}+\Delta q_{T_{3}}^{1}
\end{gathered}
$$

By using the equations (3) - (16), the average value of the input current and output current is able to be expressed as the equation (17).

$$
\begin{gathered}
\overline{I_{i n}}=\frac{\sum_{i=1}^{3} \Delta q_{T_{i}, V_{i n}}}{T}=\frac{\Delta q_{T, V_{i n}}}{T} \\
\text { and } \\
\bar{I}_{o}=\frac{\sum_{i=1}^{3} \Delta q_{T_{i}, V_{o}}}{T}=\frac{\Delta q_{T, V_{o}}}{T}
\end{gathered}
$$

By being based on the equation (17), we can see what relation there is between the current of input and output. The relation is shown as equation (18). From equations (18), it is possible to know the value of $n$ in Fig. 4; otherwise, $n$ 's value is the $n$ of ' $n$-th times'.

$$
\begin{gathered}
\overline{I_{l n}}=-k \overline{I_{o}}, \\
\text { where } \Delta q_{T_{i}, V_{i n}}=-k \Delta q_{T, V_{o}} \quad(k=1, \cdots, 12)
\end{gathered}
$$

Next step is the analysis of the consumed energy from the input power in theoretical way so that we can know the value of $R_{s c} . R_{s c}$ will be used for deriving the efficiency of the converter. $R_{s c}$ is derived by calculating how much a component consumes electronic energy.

By using equation (19), deriving the value of the $R_{s c}$ is possible through the comparison of the consumed energy in four-terminal equivalent model in Fig. 4. The consumed energy of the $R_{s c}$ in Fig. 4 is shown at equation (20) and the result of the calculation for each step is presented in table 5 .

$$
\begin{aligned}
& P=I \cdot V=I^{2} \cdot R=\frac{V^{2}}{R} \\
& W_{T}=\left(\frac{\Delta q_{V_{o}}}{T}\right)^{2} \cdot R_{S C} \cdot T
\end{aligned}
$$

Table 5. The value of $R_{s c}$ in each step

\begin{tabular}{|c|c|}
\hline Step & Value of $R_{s c}$ \\
\hline 1 times & $21 R_{\text {on }}$ \\
\hline 2 times & $27 R_{\text {on }}$ \\
\hline 3 times & $42 R_{\text {on }}$ \\
\hline 4 times & $48 R_{\text {on }}$ \\
\hline 5 times & $84 R_{\text {on }}$ \\
\hline 6 times & $87 R_{\text {on }}$ \\
\hline 7 times & $135 R_{\text {on }}$ \\
\hline 8 times & $174 R_{\text {on }}$ \\
\hline 9 times & $228 R_{\text {on }}$ \\
\hline 10 times & $282 R_{\text {on }}$ \\
\hline 11 times & $339 R_{\text {on }}$ \\
\hline 12 times & $405 R_{\text {on }}$ \\
\hline
\end{tabular}

Next step is to derive the efficiency of the converter by implementing the combination of the interaction equations above mentioned. Representatively, the relation of the factors in 12 times mode is shown at equation (21) in the way of the Kettenmatrix. The factors are the average current of the input and the output, the average voltage of the input and the output, the value of $R_{s c}$ and $n$.

$$
\left.\left[\overline{\overline{V_{l n}}}\right] \overline{I_{l n}}\right]=\left[\begin{array}{cc}
1 & 0 \\
12 & 0 \\
0 & 12
\end{array}\right]\left[\begin{array}{cc}
1 & 405 R_{S C}+2 R_{S C} \\
0 & 1
\end{array}\right]\left[\begin{array}{c} 
\pm \overline{V_{\text {out }}} \\
-\bar{I}_{o}
\end{array}\right]
$$

In equation (21), 2Rsc is for the full bridge in Fig. 6, 
because the full bridge has two switches. Then, we can take the efficiency $\eta$ and the relation between the output voltage and the efficiency, which is shown at equation (22). Others' efficiency is possible to derive by substituting the values in table 5 in the same way for $n$-th times mode.

$$
\begin{gathered}
\eta=\frac{R_{L}}{R_{L}+407 R_{\text {on }}} \text { and } \\
V_{\text {out }}= \pm\left(\frac{R_{L}}{R_{L}+407 R_{\text {on }}}\right) \times 12 V_{\text {in }}
\end{gathered}
$$

How to set the period for each mode is that we calculate the value $T_{m}(m=1 \sim 12)$ by using equation (23). The output with the time values for $T_{m}$ follows a sinusoidal wave form with $50 \mathrm{~Hz}$ as much approximately as possible. The period for each mode is shown on Table 6. In table 6, 23 kinds of step mean a half period in staircase sine waveform.

$$
T_{m}=\frac{\operatorname{acrsin}(12 \cdot n)}{100 \cdot \pi}, \text { where } n=\{1, \cdots 12\}
$$

Table 6. The period for each mode

\begin{tabular}{|c|c|c|}
\hline Step & Rise & Fall \\
\hline 1 times & $220.5 \mu \mathrm{s}$ & $277.5 \mu \mathrm{s}$ \\
\hline 2 times & $277.5 \mu \mathrm{s}$ & $277.5 \mu \mathrm{s}$ \\
\hline 3 times & $277.5 \mu \mathrm{s}$ & $279 \mu \mathrm{s}$ \\
\hline 4 times & $277.5 \mu \mathrm{s}$ & $277.5 \mu \mathrm{s}$ \\
\hline 5 times & $277.5 \mu \mathrm{s}$ & $277.5 \mu \mathrm{s}$ \\
\hline 6 times & $279 \mu \mathrm{s}$ & $277.5 \mu \mathrm{s}$ \\
\hline 7 times & $333 \mu \mathrm{s}$ & $333 \mu \mathrm{s}$ \\
\hline 8 times & $333 \mu \mathrm{s}$ & $388.5 \mu \mathrm{s}$ \\
\hline 9 times & $388.5 \mu \mathrm{s}$ & $390 \mu \mathrm{s}$ \\
\hline 10 times & $445.5 \mu \mathrm{s}$ & $444 \mu \mathrm{s}$ \\
\hline 11 times & $555 \mu \mathrm{s}$ & $555 \mu \mathrm{s}$ \\
\hline 12 times & & $2556 \mu \mathrm{s}$ \\
\hline
\end{tabular}

Next step is to establish how to analyze the efficiency of a staircase sine waveform with the values we derived. By using the values in Table 6 and equation (24), it is possible to calculate the efficiency in theoretical way. The equation (24) is the result of comparing the ideal voltage without loss with the voltage calculated by using the efficiency value at each step. Fig. 7 complements the idea about theoretical calculation above mentioned.

$$
\sum_{j=1}^{12} \frac{j \cdot t_{j} \cdot \eta_{j}}{j \cdot t_{j}}
$$

where $t_{j}: j-$ th times of rise or fall

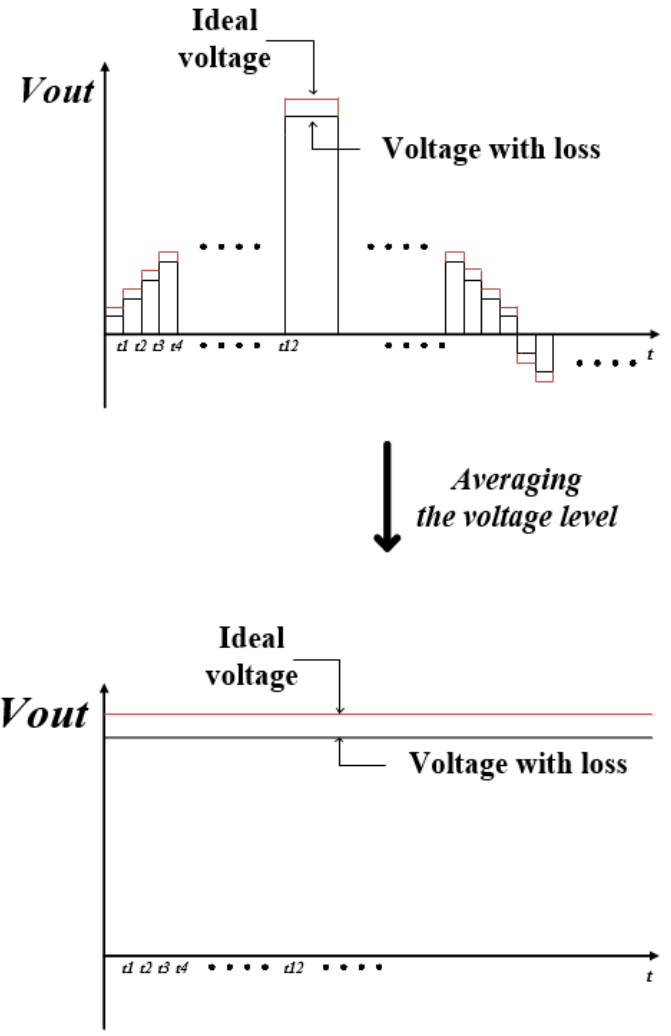

Fig. 7. Four-terminal equivalent model for the converter with all of parameters

\section{Simulation}

The simulation is implemented with the conditions shown on Table 7. The output waveform as the staircase sine waveform is shown on Fig. 8. As seeing Fig. 8, we can know that 12 kinds of step is realized showing staircase type. On Fig. 9, the power efficiency of the designed is shown. Fig. 10 illustrates the RMS of the output voltage. As Fig. 9, the error between the theoretical calculation and the simulation is $3.7 \%$ on average. The result of comparison between the theoretical calculation and the simulation is shown on Table 8 .

Table 7. Simulation conditions

\begin{tabular}{|c|c|}
\hline Parameter & Value \\
\hline$V_{\text {in }}$ & $12 \mathrm{~V}$ \\
\hline $\mathrm{C}_{1}, \mathrm{C}_{2}, \mathrm{C}_{3}, \mathrm{C}_{4}$ & $10 \mu \mathrm{F}$ \\
\hline $\mathrm{C}_{5}$ & $0.1 \mu \mathrm{F}$ \\
\hline$R_{o n}$ & $0.1 \Omega$ \\
\hline$R_{L}$ & $10 \Omega \sim 10 \mathrm{k} \Omega$ \\
\hline$T$ & $1.5 \mu \mathrm{sec}$ \\
\hline$f$ (output's frequency) & $50 \mathrm{~Hz}$ \\
\hline
\end{tabular}




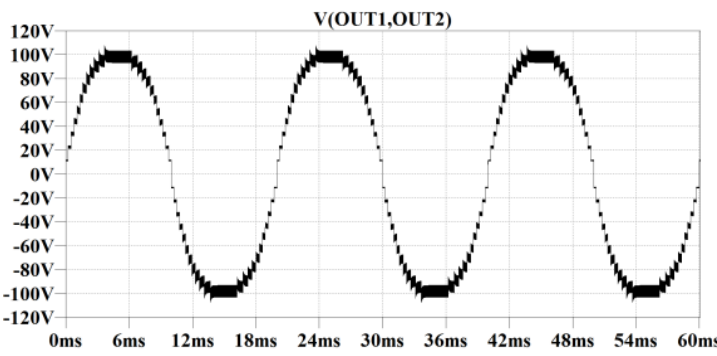

(a) $R_{L}=100 \Omega$.

V(OUT1,OUT2)

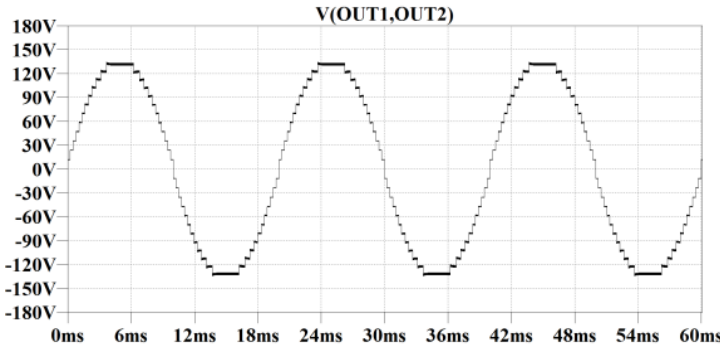

(b) $R_{L}=500 \Omega$

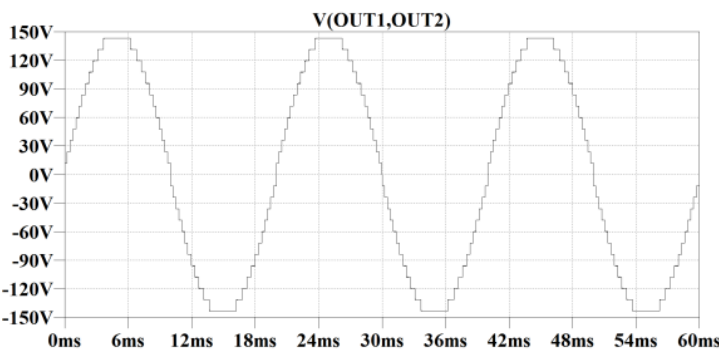

(c) $R_{L}=1000 \Omega$.

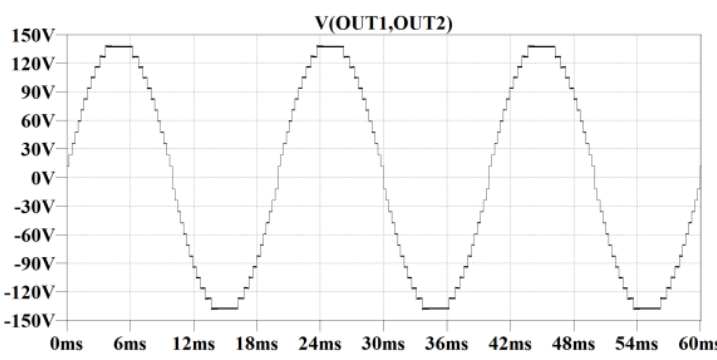

(d) $R_{L}=10000 \Omega$.

Fig. 8. Simulated output voltage with the load.

Table 8. Comparison between theoretical way and simulation.

\begin{tabular}{|c|c|c|}
\hline Load & Theoretical way & Simulation \\
\hline 10 & 32.59 & 28.58 \\
\hline 20 & 44.79 & 40.58 \\
\hline 100 & 78.48 & 73.96 \\
\hline 500 & 94.28 & 92.29 \\
\hline 1000 & 97.22 & 95.2 \\
\hline 10000 & 99.71 & 93.77 \\
\hline
\end{tabular}

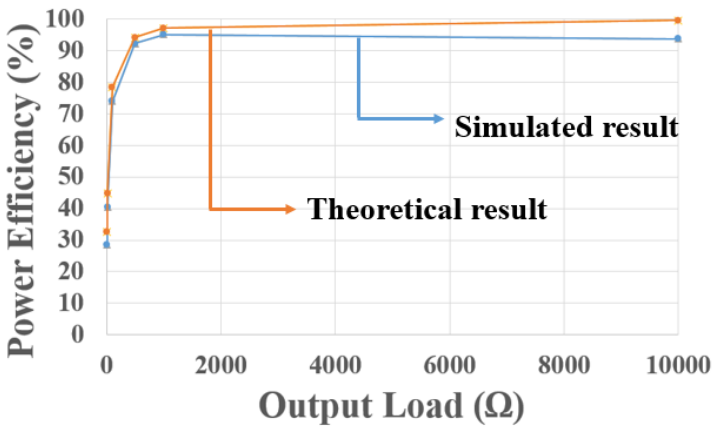

(a) Efficiency as the load increased.

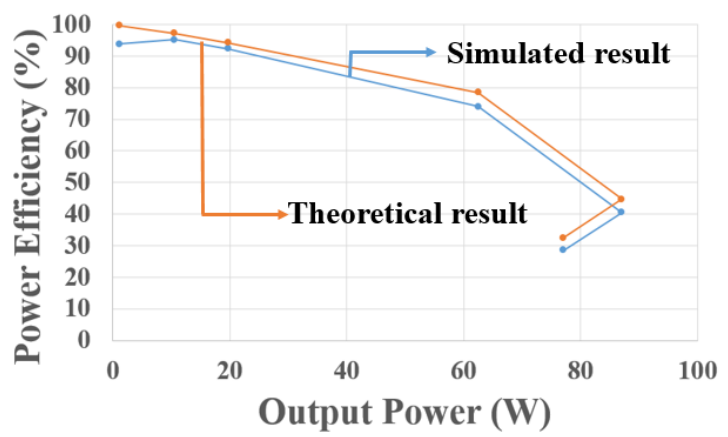

(b) Efficiency as the output power increased.

Fig. 9. Simulated and theoretical power efficiency.

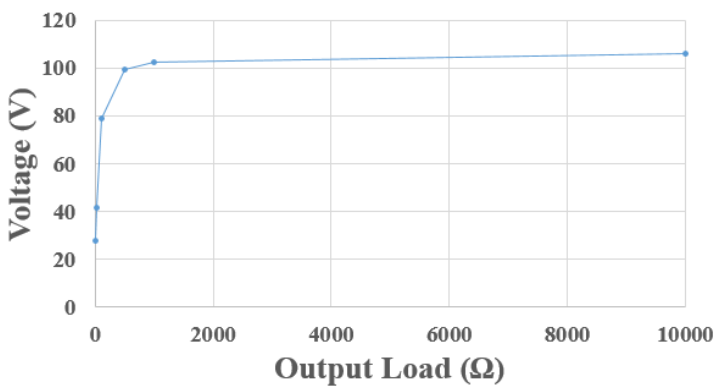

(a) $V_{\text {out }, R M S}$ as the load increased.

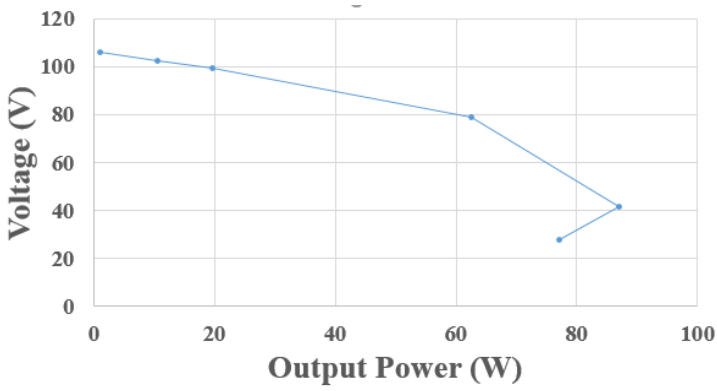

(b) $V_{\text {out }, R M S}$ as the output power increased.

Fig. 10. RMS of simulated output voltage

\section{Conclusion}

A DC-AC converter for generating a staircase sine 
waveform has been analyzed and simulated in this paper. The analysis of the converter was implemented by using a fourterminal model and mathematical way. And then we confirmed the validity of the converter by comparing the efficiency derived in theoretical way and the efficiency simulated. The error ratio was $3.7 \%$ on average. In conclusion, we proved theoretically that the designed converter is possible to generate the staircase sine waveform operated at $50 \mathrm{~Hz}$ with the validity.

The design of the prototype of the converter used in this paper is left for a future work.

\section{References}

(1) J. S. Brugler : "Theoretical performance of voltage multiplier circuits", IEEE Journal of Solid-State Circuits, Vol. 6, Issue:3, pp.132-135, 1971.

(2) M. Oota, S. Terada, K. Eguchi, and I. Oota : "Development of switched-capacitor bi-directional DCAC converter for inductive and capacitive loads", IEEE International Symposium on Industrial Electronics, pp. 1618-1623, 2009

(3) S. Terada, I. Oota, K. Eguchi, and F. Ueno : "A ring type switched-capacitor (SC) programmable converter with DC or AC input/ DC or AC output", the 47th IEEE International Midwest Symposium on Circuits and Systems, pp. I-29 - I-32, 2004.

(4) Y. H. Chang : "Modelling and analysis of multistage switched-capacitor-voltage-multiplier boost DC-AC inverter", IEEE 9th International Conference on Power Electronics and Drive Systems, pp. 523-526, 2011.

(5) K. Eguchi, P. Julsereewong, A. Julsereewong, K. Fujimoto, and H.Sasaki : "A Dickson-type adder/subtractor DC-DC converter realizing stepup/step-down conversion, Journal of Innovative Computing, Information and Control, Vol. 9, No. 1, pp. 123-138, 2013 